\title{
Gliomas: marcadores tumorais e prognóstico
}

\author{
Vinícius Trindade ${ }^{1}$, Helder Picarelli ${ }^{2}$, Eberval Gadelha Figueiredo ${ }^{3}$, Manoel Jacobsen Teixeira ${ }^{4}$ \\ Divisão de Clínica Neurocirúrgica do Hospital das Clínicas da Faculdade de Medicina da Universidade de São Paulo, \\ SP, Brasil.
}

\section{RESUMO}

Os gliomas representam 30\%-40\% de todas as neoplasias intracranianas e aproximadamente $50 \%$ são glioblastomas. São classificados em graus pela OMS, de acordo com sua patologia. Apresentam altas taxas de mortalidade. Existem marcadores tumorais que podem auxiliar na detecção precoce e avaliar prognóstico. Realizada revisão sobre o tema marcadores tumorais por meio do site PubMed. MGMT é uma proteína que restaura o DNA, impedindo a sua alquilação. A metilação do MGMT por meio de fenômeno epigenético impede sua transcrição inibindo sua ação, tornando o tumor suscetível a fármacos. IDH e codeleção cromossômica 1p19q são marcadores tumorais e estão associados a melhor prognóstico. As neoplasias intracranianas apresentam altas taxas de mortalidade e sua detecção precoce por meio de marcadores e o conhecimento de alterações que conferem bom prognóstico podem auxiliar no tratamento dessa doença. A análise molecular auxilia na detecção e no tratamento de tumores.

\section{PALAVRAS-CHAVE}

Glioma, biomarcadores farmacológicos, isocitrato desidrogenase, O(6)-metilguanina-DNA metiltransferase.

\section{ABSTRACT}

Gliomas: tumors biomarkers and prognosis

Gliomas represent $30 \%-40 \%$ of all intracranial tumors and approximately $50 \%$ are glioblastomas. They are classified by the WHO in degrees, according to their pathology. Have high mortality rates. There are tumor markers may help in early detection and assess prognosis. Was performed a review about the topic tumor markers through PubMed. MGMT is a protein that restores the DNA, preventing its alkylation. Methylation of MGMT through epigenetic phenomenon prevents their transcription and inhibits its action, making the tumor susceptible to drugs. IDH and chromosomal deletion 1p19q are tumor markers and are associated with better prognosis. The intracranial tumors have high rates of mortality and early detection through biomarkers and knowledge of changes that confer a good prognosis can help in treating this disease. Molecular analysis allows the detection and treatment of tumors.

\section{KEYWORDS}

Glioma, biomarkers pharmacological, isocitrate dehydrogenase, O(6)-methylguanine-DNA methyltransferase.

\section{Introdução}

Os gliomas representam $30 \%-40 \%$ de todas as neoplasias intracranianas. ${ }^{1}$ Aproximadamente 50\% deles são representados por glioblastomas multiformes (GBM). Em relação à distribuição etária, têm seu pico de incidência entre os 40 e 65 anos. ${ }^{1}$

Baseando-se na classificação dos tumores do SNC da Organização Mundial de Saúde (OMS), os GBMs são classificados como grau 4 por seu grau de indiferenciação, atipias, necrose e proliferação vascular, que conferem rápido crescimento, invasividade e prognósti- co reservado. A maior parte dos gliomas é esporádico e o seu principal fator de risco é a radiação iônica, sendo atribuída à hereditariedade apenas $1 \%$ a $4 \%{ }^{1}$

Apesar de não serem comuns, apresentam altas taxas de mortalidade e morbidade. $\mathrm{O}$ tratamento consiste em medidas clínicas para controle do edema cerebral e epilepsia sintomática e no tratamento específico com quimioterapia, radioterapia e neurocirurgia. ${ }^{1-3}$

Diante de tal problemática, cada vez mais se buscam meios de detecção precoce desses tumores e avaliação prognóstica, enfatizando-se nesse contexto a análise de marcadores tumorais para GBMs. ${ }^{3}$

1 Médico-residente da Neurocirurgia do Hospital das Clínicas da Faculdade de Medicina da Universidade de São Paulo (HC-FMUSP), São Paulo, SP, Brasil.

2 Neurocirurgião do Instituto do Câncer do HC-FMUSP, São Paulo, SP, Brasil.

3 Diretor da Divisão de Clínica Neurocirúrgica do HC-FMUSP, São Paulo, SP, Brasil.

4 Professor titular do Departamento de Neurocirurgia do HC-FMUSP, São Paulo, SP, Brasil. 


\section{Materiais e métodos}

Foi realizada uma revisão da literatura com pesquisa no PubMed utilizando-se os termos "gliomas", "biomarker" "MGMT", "IDH" e “deletion 1p19q". Amostragem de artigos a partir de 2004 com revisão dos marcadores MGMT, IDH e codeleção 1p19q.

\section{Resultados}

\section{Metilação MGMT}

A O6-metilguanina-DNA metiltransferase (MGMT) é uma proteína suicida que catalisa a transferência de um grupo metil da posição O6 da guanina para uma cisteína, promovendo o reparo do DNA. Atualmente, representa uma das moléculas mais estudadas em neuro-oncologia, principalmente por sua relação com prognóstico tumoral. ${ }^{4}$

O gene MGMT localiza-se no 10q26, sendo composto por 5 éxons e uma grande ilha citosina-fosfo-guanina (CPG) com 763 bases e 98 regiões CPGs, que abarca o primeiro éxon e boa parte do promotor do gene. As áreas CPGs são de importância, uma vez que nelas ocorrem fenômenos epigenéticos que regulam a expressão do gene. $\mathrm{O}$ mais comum é a metilação dessas áreas que promovem alterações impedindo a transcrição do gene, silenciando-o. Com a inexistência dessa proteína, a tendência a mutações é maior e o indivíduo tem maior suscetibilidade a sofrer de processos neoplásicos. ${ }^{4}$

No entanto, com o advento da quimioterapia com ação alquilante, observou-se que a presença da MGMT causaria a restauração do DNA da célula tumoral que sofrera metilação pelo quimioterápico, resultando em maior resistência à terapia. Assim, os indivíduos com hipermetilação do gene MGMT seriam incapazes de traduzir a proteína restauradora e, então, a ação do fármaco seria plena.

Alguns estudos mostram o potencial de bom prognóstico dessa alteração. Em análise do tratamento de indivíduos com hipermetilação do MGMT, observaram-se taxas de sobrevida em dois e cinco anos de $49 \%$ e $14 \%$, respectivamente, nos indivíduos tratados com radioterapia e quimioterapia adjuvante com termozolomide, enquanto nos indivíduos tratados apenas com radioterapia as taxas foram de $24 \%$ e $5 \%$. Nos pacientes sem a hipermetilação os resultados de sobrevida em dois a cinco anos foram de $15 \%$ a $8 \%$ nos que receberam radio e quimioterapia e de apenas $2 \%$ e $0 \%$ nos que foram tratados apenas com radioterapia. ${ }^{5-7}$
Atualmente, aventa-se a possibilidade de dividir os GBMs em dois grupos baseados no MGMT, inclusive modificando a gradação da OMS para tumores. Porém, a viabilidade de análise da proteína restauradora ainda é bastante restrita e, além disso, muitos ainda optam pelo tratamento com termozolomide, sem essa diferenciação, em decorrência de ser um fármaco oral com poucos efeitos tóxicos. ${ }^{4,8}$

Baseando-se no conceito da inativação de uma proteína restauradora, a busca por uma medicação que inative a MGMT é bastante promissora. A 06-benzilguanina (06BG) age como um potente agente inibidor da transcrição da proteína, porém sua ação também ocorre em tecidos normais, com grande toxicidade como efeito colateral. ${ }^{9}$

A hipermetilação do MGMT é um dos mais intensos fatores prognósticos, sendo observada maior sobrevida nos indivíduos que a possuem. Atualmente, sua técnica de detecção ocorre por meio de PCR, sendo limitada a grandes centros, sendo essencial a busca de novos métodos para maior difusão do exame e melhor análise da terapêutica em pacientes com essa condição. ${ }^{10}$

\section{Isocitrato desidrogenase}

A isocitrato desidrogenase (IDH) é um grupo de cinco proteínas, entre elas a IDH-1 e a IDH-2, que atuam no metabolismo oxidativo do organismo. A IDH-1 é codificada por um gene localizado no cromossomo $2 \mathrm{q} 33.3$ e está presente no citoplasma, nos peroxissomos e no retículo endoplasmático. A IDH-2 está presente apenas na mitocôndria e está localizada no 15q26.1. Ambas atuam na produção de NADPH, a partir da descarboxilação oxidativa do isocitrato a alfacetoglutarato (alfaKG), essencial para restauração da glutationa, que exercerá suas ações antioxidantes. Por sua vez, o alfaKG atuará na degradação do fator indutor de hipóxia (HIF-1alfa). ${ }^{4}$

A mutação dessa enzima promove diminuição da produção de NADPH e aumento da HIF-1alfa, que estão relacionados com efeitos pró-oncogênicos. Além disso, a alteração da enzima promove maior conversão do alfacetoglutarato em 2-hidroxi-glutarico desidrogenase (2-HG). Em pacientes com defeito associado da enzima 2-HG desidrogenase, ocorre acúmulo de 2-HG, a qual também tem efeitos pró-oncogênicos e pode também ser utilizada com marcador tumoral.,11

A mutação da IDH 1 e 2 está bastante relacionada com gliomas, principalmente os grau 2 e 3 e glioblastomas secundários, sendo presente em mais de $80 \%$ e praticamente ausentes nos demais tumores do sistema nervoso central (SNC) e demais neoplasias do organismo. Diante disso, há tendência de utilizá-lo como um marcador de glioblastomas secundários, diferenciando-os do primário, além da melhor diferenciação entre os tumores grau 1 , os quais não cursam com a mutação, e 2.,11 
Nesse contexto, observa-se, ainda, que os tumores que cursam com essa mutação têm melhor prognóstico em relação aos outros, denotando uma tendência a melhor resposta terapêutica. A explicação se basearia na provável maior suscetibilidade da célula tumoral a efeitos oxidativos, impostos pela diminuição da disponibilidade de NADPH. Portanto, pode ser uma ferramenta interessante no diagnóstico e prognóstico da doença. ${ }^{4,11}$

\section{Codeleção do cromossomo $1 p 19 q$}

A codeleção do cromossomo 1p19q revela-se como uma alteração cromossômica também relacionada com melhor prognóstico do tratamento tumoral. São alterações presentes principalmente em tumores com início em oligodendrogliócitos e estão presentes em $80 \%$ dos oligodendrogliomas tanto grau II ou III exercendo papel inicial na tumorigênese, revelando-se como um excelente marcador tumoral para diagnóstico. ${ }^{12-14}$

A alteração também está relacionada com melhor prognóstico em relação aos tumores sem a codeleção. Indivíduos com essa alteração têm maior sobrevida e progressão mais lenta do tumor. ${ }^{12-15}$

\section{Discussão}

Os gliomas são neoplasias que ainda representam grande desafio para a prática médica. Apresentam altas taxas de mortalidade e morbidade. A ideia de detecção precoce é algo bem estabelecido no contexto oncológico, determinando melhores resultados na sobrevida. As neoplasias do SNC geralmente manifestam quadro clínico em uma fase adiantada da doença, além de terem progressão rápida. Diante disso, a atual problemática consiste em, cada vez mais, encontrar fatores de detecção precoce e de bom prognóstico, antecipando o tratamento e adequando as estratégias para cada tipo de tumor. Neste artigo foram apresentados três desses fatores.

O MGMT representa uma característica bastante relacionada com melhor resposta ao efeito dos quimioterápicos alquilantes. É essencial a busca de otimizar e difundir meios para sua detecção e, assim, otimizar o direcionamento terapêutico e o prognóstico. A mutação da IDH, a 2-HG e a codeleção 1p19q podem ser utilizadas na detecção precoce, além de também serem relacionadas a melhores prognósticos em relação aos tumores que não apresentam tal deleção.

A pesquisa molecular avança cada vez mais e representa uma ferramenta principalmente no contexto neuro-oncológico. Outras moléculas também estão associadas ao prognóstico, como a betacatenina, níveis de osteopontina, CD133, entre outras. ${ }^{3,15-17}$ Esta revisão objetiva enfatizar a necessidade de direcionar os estudos para o campo molecular, que poderá ser fonte de soluções futuras.

\section{Considerações finais}

Os marcadores tumorais são um conceito moderno em neuro-oncologia e ainda existe uma vasta área a ser descoberta. Os gliomas constituem neoplasias graves com baixas taxas de cura. O campo molecular contribuirá para a melhor resolução dos casos. Novos estudos ainda são necessários.

\section{Referências}

1. Schneider T, Mawrin C, Scherlach C, Skalej M, Firsching R. Gliomas in adults. Dtsch Arztebl Int. 201;107(45):799-807.

2. Wei J, Barr J, Kong LY, Wang Y, Wu A, Sharma AK, et al. Glioblastoma cancer-initiating cells inhibit T-cell proliferation and effector responses by the signal transducers and activators of transcription 3 pathway. Mol Cancer Ther. 2010;9(1):67-78.

3. Rossi M, Magnoni L, Miracco C, Mori E, Tosi P, Pirtoli L, et al. $\beta$-catenin and Gli1 are prognostic markers in glioblastoma. Cancer Biol Ther. 2011;11(8):753-61.

4. Von Deimling A, Korshunov A, Hartmann C. The next generation of glioma biomarkers: MGMT methylation, BRAF fusions and IDH1 mutations. Brain Pathol. 2011;21(1):74-87.

5. Hegi ME, Diserens AC, Gorlia T, Hamou MF, De Tribolet $\mathrm{N}$, Weller M, et al. MGMT gene silencing and benefit from temozolomide in glioblastoma. $\mathrm{N}$ Engl $\mathrm{J}$ Med. 2005;352(10):997-1003.

6. Stupp R, Hegi ME, Mason WP, Van den Bent MJ, Taphoorn MJ, Janzer RC, et al. Effects of radiotherapy with concomitant and adjuvant temozolomide versus radiotherapy alone on survival in glioblastoma in a randomised phase III study: 5-year analysis of the EORTCNCIC trial. Lancet Oncol. 2009;10(5):459-66.

7. Stupp R, Mason WP, Van den Bent MJ, Weller M, Fisher B, Taphoorn MJ, et al. Radiotherapy plus concomitant and adjuvant temozolomide for glioblastoma. N Engl J Med. 2005;352(10):987-96.

8. Ricard D, Kaloshi G, Amiel-Benouaich A, Lejeune J, Marie Y, Mandonnet E, et al. Dynamic history of low-grade gliomas before and after temozolomide treatment. Ann Neurol. 2007;61(5):484-90.

9. Schold SC Jr, Kokkinakis DM, Chang SM, Berger MS, Hess $K R$, Schiff D, et al. O6-benzylguanine suppression of O6alkylguanine-DNA alkyltransferase in anaplastic gliomas. Neuro Oncol. 2004;6(1):28-32.

10. Hegi ME, Liu L, Herman JG, Stupp R, Wick W, Weller M, et al. Correlation of O6-methylguanine methyltransferase (MGMT) promoter methylation with clinical outcomes in glioblastoma and clinical strategies to modulate MGMT activity. J Clin Oncol. 2008;26(25):4189-99. 
11. Yen KE, Bittinger MA, Su SM, Fantin VR. Cancer-associated IDH mutations: biomarker and therapeutic opportunities. Oncogene. 2010;29(49):6409-17.

12. Ducray F, Idbaih A, De Reyniès A, Bièche I, Thillet J, Mokhtari K, et al. Anaplastic oligodendrogliomas with 1p19q codeletion have a proneural gene expression profile. Mol Cancer. 2008;7:41.

13. Cairncross G, Berkey B, Shaw E, Jenkins R, Scheithauer $B$, Brachman D, et al. Phase III trial of chemotherapy plus radiotherapy compared with radiotherapy alone for pure and mixed anaplastic oligodendroglioma: Intergroup Radiation Therapy Oncology Group Trial 9402. J Clin Oncol. 2006;24(18):2707-14.

14. Griffin CA, Burger P, Morsberger L, Yonescu R, Swierczynski $S$, Weingart JD, et al. Identification of $\operatorname{der}(1 ; 19)(q 10 ; p 10)$ in five oligodendrogliomas suggests mechanism of concurrent $1 p$ and $19 q$ loss. J Neuropathol Exp Neurol. 2006;65(10):988-94.
15. Sreekanthreddy $P$, Srinivasan $H$, Kumar DM, Nijaguna MB, Sridevi S, Vrinda M, et al. Identification of potential serum biomarkers of glioblastoma: serum osteopontin levels correlate with poor prognosis. Cancer Epidemiol Biomarkers Prev. 2010;19(6):1409-22.

16. Wang $C$, Cao S, Yan $Y$, Ying Q, Jiang T, Xu K, et al. TLR9 expression in glioma tissues correlated to glioma progression and the prognosis of GBM patients. BMC Cancer. 2010;10:415.

17. Sherman JH, Redpath GT, Redick JA, Purow BW, Laws ER, Jane JA Jr, et al. A novel fixative for immunofluorescence staining of CD133-positive glioblastoma stem cells. J Neurosci Methods. 2011;198(1):99-102.

\section{Endereço para correspondência}

Vinícius Trindade Gomes da Silva

Rua Dr. Ovídio Pires de Campos, 171, ap. 203.

05403-010 - São Paulo, SP, Brasil

Telefone: (11) 7379-8844

E-mail: viniciustrindade@hotmail.com 